\title{
Combination of ${ }^{18}$ F-FDG PET/CT and Diffusion-Weighted MR Imaging as a Predictor of Histologic Response to Neoadjuvant Chemotherapy: Preliminary Results in Osteosarcoma
}

\author{
Byung Hyun Byun ${ }^{1}$, Chang-Bae Kong ${ }^{2}$, Ilhan Lim $^{1}$, Chang Woon Choi ${ }^{1}$, Won Seok Song ${ }^{2}$, Wan Hyeong Cho ${ }^{2}$, \\ Dae-Geun Jeon ${ }^{2}$, Jae-Soo $\mathrm{Koh}^{3}$, Soo-Yong Lee ${ }^{2}$, and Sang Moo Lim ${ }^{1}$ \\ ${ }^{I}$ Department of Nuclear Medicine, Korea Cancer Center Hospital, Korea Institute of Radiological and Medical Sciences (KIRAMS), \\ Seoul, Republic of Korea; ${ }^{2}$ Department of Orthopedic Surgery, Korea Cancer Center Hospital, Korea Institute of Radiological and \\ Medical Sciences (KIRAMS), Seoul, Republic of Korea; and ${ }^{3}$ Department of Pathology, Korea Cancer Center Hospital, Korea \\ Institute of Radiological and Medical Sciences (KIRAMS), Seoul, Republic of Korea
}

We evaluated the potential of ${ }^{18} \mathrm{~F}-\mathrm{FDG} \mathrm{PET} / \mathrm{CT}$ and diffusionweighted imaging (DWI) to monitor the histologic response in patients with extremity osteosarcoma receiving neoadjuvant chemotherapy, using sequential PET/CT and MR imaging. Methods: We prospectively registered 28 patients with high-grade osteosarcoma treated with 2 cycles of neoadjuvant chemotherapy and surgery. All patients underwent sequential ${ }^{18} \mathrm{~F}-\mathrm{FDG}$ PET/CT and MR imaging before (PET/MR1) and after neoadjuvant chemotherapy (PET/MR2). Maximum standardized uptake value (SUV), tumor volume based on MR imaging (MRV), and the mean apparent diffusion coefficient (ADC) values were measured on PET/MR1 (SUV1, MRV1, and ADC1) and PET/MR2 (SUV2, MRV2, and ADC2). The percentage changes in maximum SUV ( $\triangle S U V), M R V(\triangle M R V)$, and ADC $(\triangle A D C)$ were calculated, and the correlations among these parameters were evaluated. After surgery, the effects of neoadjuvant chemotherapy were graded histopathologically: grades III and IV (necrosis of $\geq 90 \%$ ) indicated a good response, and grades I and II (necrosis of $<90 \%$ ) indicated a poor response. The optimum cutoff values of $\triangle S U V, \triangle M R V, \triangle A D C$, and their combination for predicting histologic response were assessed by single- and multi-receiver-operating-characteristic curve analysis. Results: Twenty-seven patients were enrolled in the present study after 1 patient with inadequate acquisition of MR imaging was excluded. $\triangle S U V$ and $\triangle A D C$ negatively correlated with each other $(\rho=-0.593$, $P=0.001$ ), and $\triangle \mathrm{MRV}$ did not correlate with $\triangle \mathrm{SUV}$ or $\triangle \mathrm{ADC}$. The cutoff value, sensitivity, specificity, and accuracy for predicting good histologic response were $\leq-52 \%, 67 \%, 87 \%$, and $78 \%$, respectively, for $\Delta$ SUV and $>13 \%, 83 \%, 73 \%$, and $78 \%$, respectively, for $\triangle \mathrm{ADC}$. However, $\triangle \mathrm{MRV}$ did not predict histologic response. Sensitivity, specificity, and accuracy were $83 \%, 87 \%$, and $85 \%$, respectively, using the combined criterion of $\triangle \mathrm{SUV} \leq-31 \%$ and $\triangle A D C>13 \%$. Conclusion: In the current preliminary study, both $\mathrm{PET} / \mathrm{CT}$ and DWI are useful for predicting histologic response after neoadjuvant chemotherapy in osteosarcoma. Combining PET/CT and

Received Oct. 19, 2012; revision accepted Jan. 29, 2013.

For correspondence or reprints contact: Sang Moo Lim, Department of Nuclear Medicine, Korea Cancer Center Hospital, Korea Institute of Radiological and Medical Sciences (KIRAMS), 75 Nowongil, Nowon Gu, Seoul, 139-706, Republic of Korea.

E-mail: smlim328@kcch.re.kr

Published online May 13, 2013.

COPYRIGHT (C) 2013 by the Society of Nuclear Medicine and Molecular Imaging, Inc.
DWI may be an effective method to predict the histologic response of patients to neoadjuvant chemotherapy.

Key Words: ADC; PET; MR imaging; osteosarcoma; chemotherapy

J Nucl Med 2013; 54:1053-1059

DOI: 10.2967/jnumed.112.115964

A djuvant and neoadjuvant chemotherapy have significantly improved the long-term survival rate of patients with high-grade osteosarcoma, compared with surgery alone (1). The current standard of chemotherapy response evaluation is to histologically assess the tumor necrosis of the excised lesion $(2,3)$, which has been reported to be the most important prognostic factor in osteosarcoma after neoadjuvant chemotherapy (4). However, because tumor necrosis can be assessed only in the resected specimens after the completion of neoadjuvant chemotherapy, the continuation of ineffective chemotherapy can cause the development of resistant clones (3). To overcome these limitations, noninvasive imaging modalities including bone scintigraphy (5), CT (6), MR imaging $(2,3,7,8)$, and ${ }^{18}$ F-FDG PET $(9-11)$ have been investigated to predict the effect of neoadjuvant chemotherapy.

${ }^{18} \mathrm{~F}-\mathrm{FDG}$ PET enables the assessment of glucose metabolism and hence also the metabolic activity of cancer tissue by calculating a standardized uptake value (SUV). Change in SUV after neoadjuvant chemotherapy has been reported to be useful in predicting tumor response in osteosarcoma (9-11). Diffusion-weighted MR imaging (DWI) represents in vivo images of biologic tissues weighted with the local microstructural characteristics of water diffusion. The apparent diffusion coefficient (ADC) is used to quantify water diffusion and tends to decrease in highly cellular tissue such as malignant tumors (12). Change in ADC value after neoadjuvant chemotherapy also has been reported to be useful in monitoring therapeutic response of osteosarcoma $(2,3,7,8)$. To our knowledge, however, there is no published study comparing the response assessment of ${ }^{18} \mathrm{~F}-\mathrm{FDG}$ PET and DWI in patients with osteosarcoma.

Recently, combined PET/MR imaging systems have been shown to allow simultaneous (13) or sequential acquisition (14) 
of PET and MR images, and literature describing the initial clinical experiences with PET/MR imaging in oncology is already available $(15,16)$. In this prospective study, we evaluated the potential of ${ }^{18} \mathrm{~F}$-FDG PET/CT and DWI to monitor the histologic response in patients with extremity osteosarcoma receiving neoadjuvant chemotherapy using sequential PET/CT and MR imaging.

\section{MATERIALS AND METHODS}

\section{Patients and Treatment}

The primary aim of the current preliminary study was to assess the diagnostic accuracy of the combination of ${ }^{18}$ F-FDG PET/CT and DWI, compared with each modality alone, in the prediction of histologic response after neoadjuvant chemotherapy for osteosarcoma. The secondary aim of the study was to assess the correlations among the changes in maximum SUV (SUVmax), tumor volume, and ADC value after neoadjuvant chemotherapy.

Eligibility requirements included newly diagnosed histopathologically proven primary high-grade extremity osteosarcoma, the completion of neoadjuvant chemotherapy and surgery, sequential ${ }^{18} \mathrm{~F}$-FDG $\mathrm{PET} / \mathrm{CT}$ and MR imaging scans obtained before (PET/MR1) and after the completion of neoadjuvant chemotherapy (PET/MR2), a time lapse between PET/MR1 and the initiation of neoadjuvant chemotherapy of no more than $2 \mathrm{wk}$, and a time lapse between PET/MR2 and surgery of no more than $2 \mathrm{wk}$ (10). Twenty-eight consecutive patients were prospectively registered between May 2010 and March 2012. One patient with inadequate acquisition of MR images after neoadjuvant chemotherapy due to motion artifact was excluded. Thus, 27 patients were enrolled in the present study.

All patients received 2 cycles of neoadjuvant chemotherapy with a combination of high-dose methotrexate at a dose of $8-12 \mathrm{~g} / \mathrm{m}^{2}$, Adriamycin (Pharmachemie BV) at a dose of $60 \mathrm{mg} / \mathrm{m}^{2}$, and cisplatin at a dose of $100 \mathrm{mg} / \mathrm{m}^{2}$, using the modified $\mathrm{T} 10$ protocol (17). This study was approved by our institutional review board. All patients provided written informed consent, and this study was performed according to the ethical guidelines of our institutional clinical research committee.

\section{Protocol of Sequential ${ }^{18}$ F-FDG PET/CT and MR Imaging}

The whole-body PET/CT scanner (Biograph 6; Siemens Medical Solutions) was placed parallel to the 3.0-T whole-body MR imaging scanner (MAGNETOM Trio A Tim; Siemens Medical Solutions). These 2 scanners were located $10 \mathrm{~m}$ apart, separated by an antimagnetic wall. PET/CT imaging from the vertex to the upper thigh (5-6 bed positions) was performed $60 \mathrm{~min}$ after ${ }^{18} \mathrm{~F}-\mathrm{FDG}$ injection, and this was followed by additional PET/CT scanning of the lower extremities (6-7 bed positions). Then, MR images covering the tumor sites were acquired immediately after the completion of PET/CT scanning. Both $\mathrm{PET} / \mathrm{CT}$ and MR images were acquired with the patient supine, and all patients moved from the PET/CT scanner to the MR imaging scanner (on independent patient tables) by walking.

\section{F-FDG PET/CT Acquisition}

All patients fasted for at least $6 \mathrm{~h}$ before the scans, and blood glucose levels did not exceed $7.2 \mathrm{mmol} / \mathrm{L}$ in any patient. We administered $7.4 \mathrm{MBq} / \mathrm{kg}$ of body weight of ${ }^{18} \mathrm{~F}-\mathrm{FDG}$ in the case of children $(<15 \mathrm{y}$ of age). Otherwise, we administered of $370 \mathrm{MBq}$ of ${ }^{18} \mathrm{~F}-\mathrm{FDG}$ irrespective of body weight. Immediately after the $\mathrm{CT}$ acquisition without intravenous iodinated contrast, PET data were acquired in the same anatomic locations, with a $16.2-\mathrm{cm}$ axial field of view in 3-dimensional mode at $210 \mathrm{~s}$ per bed position. CT images were obtained using a 6-slice helical CT scanner, and the imaging parameters used for CT scans were as follows: $130 \mathrm{kVp}, 30 \mathrm{~mA}, 0.6$ s per CT rotation, and a pitch of 6 . The CT data were used for attenuation correction, and the images were reconstructed using a conventional iterative algorithm (ordered-subsets expectation-maximization, 2 iterations, and 8 subsets). The same acquisition processes were performed in both PET/ CT scans (from skull base to upper thigh and extremity).

\section{MR Imaging Acquisition}

MR images were obtained using a body array surface coil. MR imaging included a standard T1-weighted sequence (repetition time [TR]/echo time [TE], 752/10 ms; field of view, $450 \mathrm{~mm}$; matrix size, $279 \times 448$; slice thickness, $5 \mathrm{~mm}$; 2 average; acquisition time, $3 \mathrm{~min}$ $53 \mathrm{~s}$ ), with or without gadolinium enhancement, and a T2-weighted sequence (TR/TE, 3,360/97 ms; field of view, $450 \mathrm{~mm}$; matrix size, $298 \times 448$; slice thickness, $5 \mathrm{~mm}$; 2 average; acquisition time, $3 \mathrm{~min}$ $7 \mathrm{~s}$ ), without fat suppression. The contrast agent, Gd-DTPA-BMA (generic name, gadodiamide; Omniscan [GE Healthcare]), was injected intravenously as a bolus $(0.1 \mathrm{mmol} / \mathrm{kg})$ at a rate of 1.5 $\mathrm{mL} / \mathrm{s}$ using a power injector. This injection was followed by a $20-$ $\mathrm{mL}$ saline flush. Diffusion-weighted images were acquired using a spin-echo-type single-shot echo-planner imaging sequence. The parameters used were as follows: TR/TE, 6,100/78 ms; field of view, $380 \mathrm{~mm}$; matrix size, $100 \times 128$; slice thickness, $4 \mathrm{~mm}$; receiver bandwidth, 3,004 Hz/pixel; and acquisition time of 4 min $22 \mathrm{~s}$. Imaging was performed with $b$ values of 0 and $800 \mathrm{~s} / \mathrm{mm}^{2}$. Diffusion images were integrated in the 3 orthogonal directions to calculate the ADC maps.

\section{Image Analysis}

Sequential ${ }^{18} \mathrm{~F}-\mathrm{FDG}$ PET/CT and MR images were reviewed on the e-soft workstations (Siemens Medical Systems). We drew an ellipsoid volume of interest including the entire primary tumor of the extremity and measured the SUVmax on each PET/CT dataset. All PET/CT scans were reviewed and interpreted by an experienced nuclear medicine physician. Intramedullary tumor lengths were measured in coronal sections of unenhanced T1-weighted sequences; tumor widths and depths were measured in enhanced axial T1-weighted sequences without fat suppression (18). MR images were independently reviewed by 2 of the authors. When these 2 reviewers found a size discrepancy of more than $10 \%$, images were reviewed simultaneously and decisions were made by consensus. Then, the tumor volume based on MR images (MRV) was calculated from the tumor length, width, and depth using the ellipsoid formula (19). To measure the mean ADC values, we selected an axial slice with the largest tumor diameter from the ADC map and drew a freehand region of interest by referring to the enhancing solid portion of enhanced axial T1-weigted images. Two of the authors, by consensus, carefully drew the regions of interest inside the tumor, avoiding the cystic portion or visual artifact. The mean ADC values within each region of interest were recorded.

\section{Definitions of Parameters}

To assess the changes of SUVmax, MRV, and ADC values after neoadjuvant chemotherapy, the SUVmax of PET/MR1 (SUV1), the SUVmax of PET/MR2 (SUV2), the tumor volume of PET/MR1 (MRV1), the tumor volume of PET/MR2 (MRV2), the ADC value of PET/MR1 (ADC1), and the ADC value of PET/MR2 (ADC2) were measured and the following parameters were calculated using these values:

$$
\begin{gathered}
\Delta \operatorname{SUV}(\%)=(\mathrm{SUV} 2-\mathrm{SUV} 1) \times 100 / \mathrm{SUV} 1 \\
\Delta \mathrm{MRV}(\%)=(\mathrm{MRV} 2-\mathrm{MRV} 1) \times 100 / \mathrm{MRV} 1 \\
\Delta \mathrm{ADC}(\%)=(\mathrm{ADC} 2-\mathrm{ADC} 1) \times 100 / \mathrm{ADC} 1
\end{gathered}
$$




\section{Histologic Analysis}

Histologic responses to neoadjuvant chemotherapy were graded as III and IV (tumor necrosis $\geq 90 \%$ ), indicating a good response, or as I and II (necrosis $<90 \%$ ), indicating a poor response (20).

\section{Statistical Analysis}

We presented statistics as the frequencies and percentages for categoric variables or as both the mean $\pm \mathrm{SD}$ and the median (interquartile range $[\mathrm{IQR}]$ ) for continuous variables. All continuous variables were tested for normal distribution using the Shapiro-Wilk test. Tumor volume before neoadjuvant chemotherapy was compared between the patients with good histologic response and poor histologic response using the Mann-Whitney test. MRV, SUVmax, and ADC values before and after neoadjuvant chemotherapy were compared according to the histologic response (good and poor responses) using the Student $t$ test for paired data for normally distributed variables and the Wilcoxon test for nonnormally distributed variables. The correlation between percentage change in SUV ( $\triangle \mathrm{SUV})$ and ADC $(\triangle \mathrm{ADC})$ was evaluated using the Spearman correlation coefficient. We analyzed the predictive values of $\triangle \mathrm{MRV}, \triangle \mathrm{SUV}$, and $\triangle \mathrm{ADC}$ in terms of their abilities to distinguish good responders from poor responders. For this purpose, we used receiveroperating-characteristic (ROC) curves and calculated areas under the curves (AUCs) for each parameter. Then, the combinations of parameters that distinguished good responders from poor responders were tested by multi-ROC curve analysis, where the ROC curve was plotted on the basis of the following rule: "Each component is fixed at a diagnostic threshold except for one threshold, which is varied over all of its possible values, and the corresponding sensitivities and false-positive rates are plotted" (21). On the basis of the ROC curve analysis, the optimal criteria of $\triangle \mathrm{MRV}, \triangle \mathrm{SUV}, \triangle \mathrm{ADC}$, and their combinations that offered the highest sum of sensitivity and specificity for predicting good response were determined. With these optimal criteria, we calculated the sensitivity, specificity, positive predictive value (PPV), negative predictive value (NPV), and accuracy for predicting good histologic response. The statistical significance of the difference among the areas under 2 or more ROC curves (95\% confidence intervals [CIs]) was calculated using the method proposed by Hanley and McNeil (22). Diagnostic performances were compared using the McNemar test. The statistical tests were performed using SPSS (version 13.0; SPSS Inc.) and MedCalc (MedCalc Software). All $P$ values were 2 sided, and a value of less 0.05 was considered statistically significant.

\section{RESULTS}

\section{Patient Characteristics}

The characteristics of all patients are detailed in Table 1 . The mean and median ages were $20.6 \pm 12.6$ and 15 y (IQR, 14-23 y), respectively, and more than half the patients $(56 \%)$ were men. According to the revised American Joint Committee on Cancer staging system, about half of the patients (52\%) were stage IIA and $15 \%$ of patients had skip or distant metastasis at diagnosis. The most frequent location of the primary tumor was the femur $(60 \%)$, followed by the tibia $(33 \%)$. The histologic subtype was osteoblastic in most patients $(85 \%)$. The mean and median time interval between the end of neoadjuvant chemotherapy and PET/MR2 were $12.8 \pm 6.0$ and $14 \mathrm{~d}$ (IQR, 11-16 d), respectively, and the mean and median time interval between the PET/MR2 and surgery were $3.0 \pm 4.8$ and $2 \mathrm{~d}$ (IQR, 1-3 d), respectively.

\section{Imaging Performance}

Twelve patients (44\%) showed a good histologic response in the resected specimens after neoadjuvant chemotherapy. The median
TABLE 1

Patient Characteristics

\begin{tabular}{|c|c|}
\hline Characteristic & Value \\
\hline \multicolumn{2}{|l|}{ Age $(y)$} \\
\hline$\leq 15$ & $14(51.9 \%)$ \\
\hline$>15$ and $\leq 40$ & $10(37.0 \%)$ \\
\hline$>40$ & $3(11.1 \%)$ \\
\hline \multicolumn{2}{|l|}{$\operatorname{Sex}(n)$} \\
\hline Male & 15 (55.6\%) \\
\hline Female & $12(44.4 \%)$ \\
\hline \multicolumn{2}{|c|}{$\begin{array}{l}\text { American Joint Committee on } \\
\text { Cancer stage }(n)\end{array}$} \\
\hline IIA & $14(51.9 \%)$ \\
\hline IIB & $9(33.3 \%)$ \\
\hline III & $1(3.7 \%)$ \\
\hline IVA & $1(3.7 \%)$ \\
\hline IVB & $2(7.4 \%)$ \\
\hline \multicolumn{2}{|c|}{ Location of primary tumor $(n)$} \\
\hline Femur & $16(59.3 \%)$ \\
\hline Tibia & 9 (33.3\%) \\
\hline Fibula & $1(3.7 \%)$ \\
\hline Radius & $1(3.7 \%)$ \\
\hline \multicolumn{2}{|l|}{ Pathologic subtype (n) } \\
\hline Osteoblastic & $23(85.2 \%)$ \\
\hline Fibroblastic & $3(11.1 \%)$ \\
\hline Telangiectatic & $1(3.7 \%)$ \\
\hline \multicolumn{2}{|l|}{$\operatorname{MRV} 1\left(\mathrm{~cm}^{3}\right)$} \\
\hline Mean $\pm S D$ & $130 \pm 108$ \\
\hline Median & 94 \\
\hline IQR & $40-223$ \\
\hline
\end{tabular}

MRV1 of the 27 osteosarcomas was $94.5 \mathrm{~cm}^{3}$ (IQR, 40.6-223.4 $\mathrm{cm}^{3}$; mean $\pm \mathrm{SD}, 130.6 \pm 108.8 \mathrm{~cm}^{3}$ ), and no significant difference in the median MRV1 was observed between patients with a good histologic response (median, $49.4 \mathrm{~cm}^{3}$; IQR, 38.8-140.3 $\mathrm{cm}^{3}$; mean $\left.\pm \mathrm{SD}, 130.8 \pm 100.5 \mathrm{~cm}^{3}\right)$ and patients with a poor histologic response (median, $116.9 \mathrm{~cm}^{3}$; IQR, 74.4-246.0 $\mathrm{cm}^{3}$; mean $\left.\pm \mathrm{SD}, 152.0 \pm 113.8 \mathrm{~cm}^{3}\right)(P=0.399)$.

After the completion of neoadjuvant chemotherapy, all patients had tumors that demonstrated ${ }^{18} \mathrm{~F}$-FDG uptake greater than adjacent normal bone. The mean SUVmax of the 27 patients decreased from $9.2 \pm 6.0$ (median, 7.7; IQR, 5.6-10.3) to $5.4 \pm 3.5$ (median, 4.2; IQR, 2.8-7.0) $(P=0.002)$, and the median ADC value increased from $1.346 \times 10^{-3} \mathrm{~mm}^{2} / \mathrm{s}$ (IQR, $1.201-1.551 \times 10^{-3}$ $\mathrm{mm}^{2} / \mathrm{s} ;$ median $\left.\pm \mathrm{SD}, 1.336 \pm 0.226 \times 10^{-3} \mathrm{~mm}^{2} / \mathrm{s}\right)$ to $1.612 \times$ $10^{-3} \mathrm{~mm}^{2} / \mathrm{s}$ (IQR, $1.290-1.809 \times 10^{-3} \mathrm{~mm}^{2} / \mathrm{s}$; median $\pm \mathrm{SD}$, $\left.1.568 \pm 0.305 \times 10^{-3} \mathrm{~mm}^{2} / \mathrm{s}\right)(P=0.002)$ after neoadjuvant chemotherapy. However, there was no significant difference between the median MRV1 (median, $94.5 \mathrm{~cm}^{3}$; IQR, 40.6-223.4 $\mathrm{cm}^{3}$; mean $\pm \mathrm{SD}, 130.6 \pm 108.8 \mathrm{~cm}^{3}$ ) and the median MRV2 (median, 103.1 $\mathrm{cm}^{3}$; IQR, 33.1-191.7 $\mathrm{cm}^{3}$; mean $\left.\pm \mathrm{SD}, 125.7 \pm 114.5 \mathrm{~cm}^{3}\right)(P=$ 0.700). In a subgroup of patients with a good histologic response, the mean SUVmax decreased from $7.5 \pm 3.1$ (median, 6.9; IQR, 5.010.2) to $3.4 \pm 1.4$ (median, 2.8; IQR, 2.4-4.2) $(P<0.001)$, and the median ADC value increased from $1.276 \times 10^{-3} \mathrm{~mm}^{2} / \mathrm{s}$ (IQR, $1.203-1.529 \times 10^{-3} \mathrm{~mm}^{2} / \mathrm{s}$; mean $\pm \mathrm{SD}, 1.333 \pm 0.183 \times 10^{-3}$ $\mathrm{mm}^{2} / \mathrm{s}$ ) to $1.704 \times 10^{-3} \mathrm{~mm}^{2} / \mathrm{s}$ (IQR, $1.484-1.821 \times 10^{-3} \mathrm{~mm}^{2} / \mathrm{s}$; mean $\left.\pm \mathrm{SD}, 1.666 \pm 0.229 \times 10^{-3} \mathrm{~mm}^{2} / \mathrm{s}\right)(P=0.012)$ after neoadjuvant chemotherapy. However, there was no significant difference between the median MRV1 (median, $49.4 \mathrm{~cm}^{3}$; IQR, 38.8-140.3 $\mathrm{cm}^{3}$; mean $\pm \mathrm{SD}, 103.8 \pm 100.5 \mathrm{~cm}^{3}$ ) and the median MRV2 


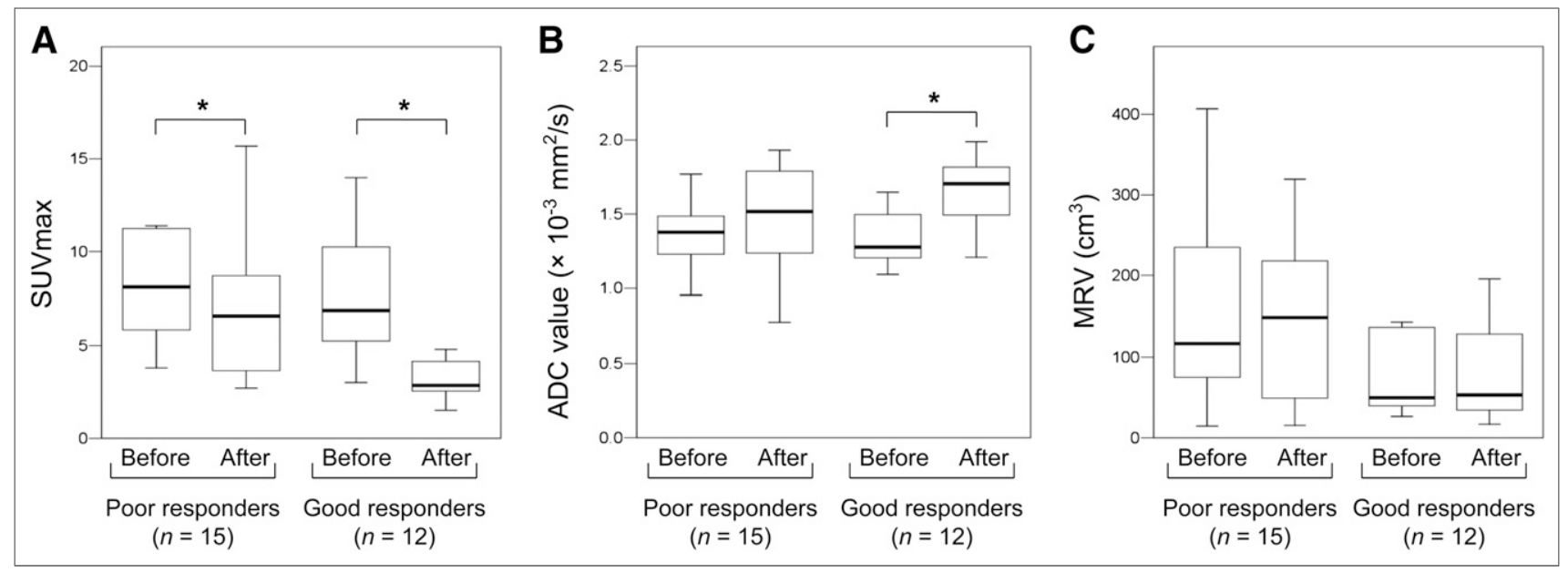

FIGURE 1. SUVmax (A), ADC value (B), and MRV (C) changes after neoadjuvant chemotherapy of poor and good histologic responders. Data are presented as box plots indicating median and IQR; whiskers extend to most extreme data points up to 1.5 times IQR. ${ }^{\star} P<0.05$ (Student $t$ test for paired data for SUVmax and Wilcoxon test for ADC value and MRV, respectively).

(median, $53.2 \mathrm{~cm}^{3}$; IQR, 32.0-139.5 $\mathrm{cm}^{3}$; mean $\pm \mathrm{SD}, 82.7 \pm 64.2$ $\left.\mathrm{cm}^{3}\right)(P=0.700)$. In another subgroup of patients with a poor histologic response, the mean SUVmax decreased from $10.6 \pm 7.5$ (median, 8.1; IQR, 5.8-11.4) to $7.1 \pm 3.9$ (median, 6.6; IQR, 3.19.4) $(P=0.018)$ after neoadjuvant chemotherapy. However, there was no significant difference between the median ADC1 (median, $1.378 \times 10^{-3} \mathrm{~mm}^{2} / \mathrm{s}$; IQR, $1.177-1.551 \times 10^{-3} \mathrm{~mm}^{2} / \mathrm{s}$; mean $\pm \mathrm{SD}$, $1.338 \pm 0.262 \times 10^{-3} \mathrm{~mm}^{2} / \mathrm{s}$ ) and the median ADC2 (median, 1.518 $\times 10^{-3} \mathrm{~mm}^{2} / \mathrm{s} ;$ IQR, $1.220-1.793 \times 10^{-3} \mathrm{~mm}^{2} / \mathrm{s}$; mean $\pm \mathrm{SD}, 1.489$ $\left.\pm 0.342 \times 10^{-3} \mathrm{~mm}^{2} / \mathrm{s}\right)(P=0.088)$, or between the median MRV1 (median, $116.9 \mathrm{~cm}^{3}$; IQR, 74.4-246.0 $\mathrm{cm}^{3}$; mean $\pm \mathrm{SD}, 152.0 \pm$ $113.8 \mathrm{~cm}^{3}$ ) and the median MRV2 (median, $149.3 \mathrm{~cm}^{3}$; IQR, 33.1$243.2 \mathrm{~cm}^{3}$; mean $\left.\pm \mathrm{SD}, 160.2 \pm 135.2 \mathrm{~cm}^{3}\right)(P=0.609)$ (Fig. 1$)$. The $\triangle$ SUVs and $\triangle$ ADCs of the 27 patients were inversely correlated ( $\rho=-0.593, P=0.001$ ) (Fig. 2). However, there was no significant correlation between $\triangle \mathrm{MRVs}$ and $\triangle \mathrm{SUVs}(P=0.8206)$ or between $\triangle \mathrm{MRVs}$ and $\triangle \mathrm{ADCs}(P=0.4026)$.

All parameters, with the exception of $\triangle \mathrm{MRV}$, predicted histologic response based on ROC curve analyses. The area under the ROC curve of $\triangle \mathrm{SUV}$ for predicting a good histologic response (AUC, 0.772; 95\% CI, 0.571-0.910; $P=0.0037$ ) was higher than those of $\triangle \mathrm{MRV}$ (AUC, 0.656; 95\% CI, 0.449-0.826; $P=0.157$ ) and $\triangle \mathrm{ADC}$ (AUC, $0.728 ; 95 \% \mathrm{CI}, 0.523-0.880 ; P=0.0338$ ), but there was no significant difference between them $(P=0.36$ between $\triangle \mathrm{SUV}$ and $\triangle \mathrm{MRV}$ and $P=0.665$ between $\triangle \mathrm{SUV}$ and $\triangle \mathrm{ADC}$ ). The optimal criteria for distinguishing a good histologic response from a poor histologic response in the ROC curve analysis were $\triangle \mathrm{SUV} \leq-52 \%$ and $\triangle \mathrm{ADC}>13 \%$, respectively. $\triangle \mathrm{SUV}$ showed $67 \%$ sensitivity, $87 \%$ specificity, $80 \%$ PPV, $76 \%$ NPV, and $78 \%$ accuracy, and $\triangle \mathrm{ADC}$ showed $83 \%$ sensitivity, $73 \%$ specificity, $71 \% \mathrm{PPV}, 85 \% \mathrm{NPV}$, and $78 \%$ accuracy for predicting good histologic response using these criteria (Fig. 3; Table 2).

\section{Combined Use of $\triangle S U V$ and $\triangle A D C$}

For the combined use of $\triangle \mathrm{SUV}$ and $\triangle \mathrm{ADC}$ to assess the histologic response after neoadjuvant chemotherapy, $\triangle \mathrm{SUV} \leq-31 \%$ and $\triangle \mathrm{ADC}>13 \%$ was chosen as the best criterion because this combination yielded the highest sum of sensitivity and specificity among all possible combinations in multi-ROC analysis. With this combined criterion, the AUC (AUC, 0.850; 95\% CI, 0.660-0.957) was higher than that of either $\triangle \mathrm{SUV}$ or $\triangle \mathrm{ADC}$ used alone, but there was no statistically significant difference (Fig. 4). The combined use of $\triangle \mathrm{SUV}$ and $\triangle \mathrm{ADC}$ showed $83 \%$ sensitivity, $87 \%$ specificity, $83 \%$ PPV, $87 \% \mathrm{NPV}$, and $85 \%$ accuracy in the prediction of good histologic response, but there was no significant difference, compared with $\triangle \mathrm{SUV}$ or $\triangle \mathrm{ADC}$ used alone (Table 2).

\section{DISCUSSION}

This is the first study, to our knowledge, measuring both $\triangle \mathrm{SUV}$ and $\triangle \mathrm{ADC}$ of osteosarcoma after the completion of neoadjuvant chemotherapy using sequential ${ }^{18}$ F-FDG PET/CT and MR imaging. $\triangle \mathrm{SUV}$ and $\triangle \mathrm{ADC}$ negatively correlated with each other, and both parameters predicted histologic response after neoadjuvant chemotherapy. The combined use of $\triangle \mathrm{SUV}$ and $\triangle \mathrm{ADC}$ served to discriminate good histologic responses.

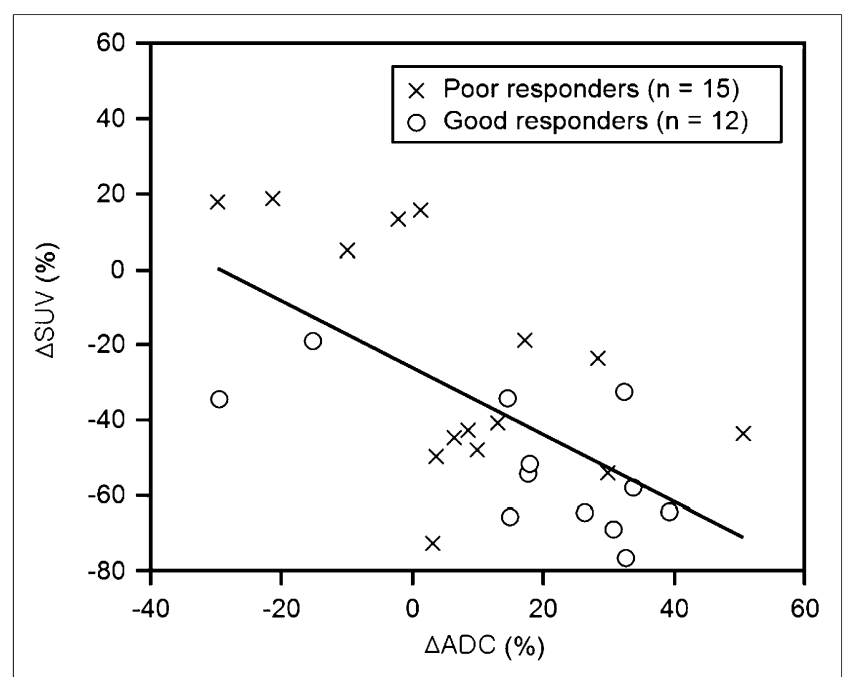

FIGURE 2. Scatterplot showing relationship between $\triangle A D C$ and $\triangle S U V$ after neoadjuvant chemotherapy. $\triangle A D C$ negatively correlated with $\Delta \operatorname{SUV}(\rho=-0.593, P=0.001)$. 

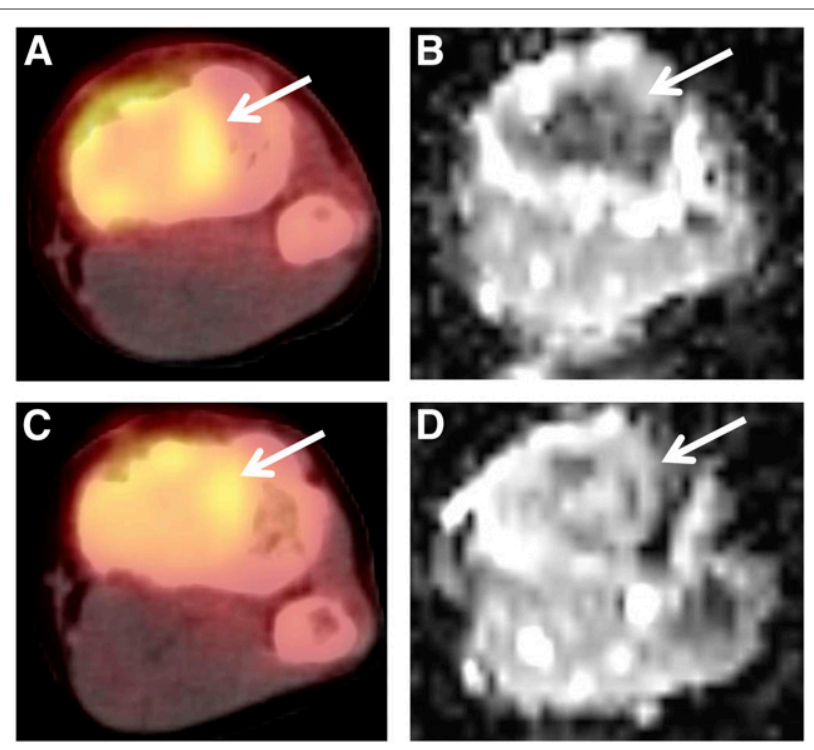

FIGURE 3. A 15-y-old male patient with osteosarcoma of left tibia who showed good histologic response after completing neoadjuvant chemotherapy. Although patient was classified as poor responder based on $\triangle S U V$, he was classified as good responder based on $\triangle A D C$ or combined use of $\triangle S U V$ and $\triangle A D C$. Baseline transverse PET/CT image (A) showed increased ${ }^{18} \mathrm{~F}-\mathrm{FDG}$ uptake of tumor with SUVmax of 2.9. Postchemotherapy PET/CT image (C) showed residual ${ }^{18} \mathrm{~F}-\mathrm{FDG}$ uptake with SUVmax of 2.0; thereby, $\triangle$ SUV was $-31.0 \%$. In baseline ADC map (B), mean ADC of tumor was $1.092 \times 10^{-3} \mathrm{~mm}^{2} / \mathrm{s}$. In postchemotherapy ADC map (D), mean ADC was $1.612 \times 10^{-3} \mathrm{~mm}^{2} / \mathrm{s}$; thereby, $\triangle \mathrm{ADC}$ was $47.6 \%$.

It has been reported that $\triangle \mathrm{MRV}$ s after neoadjuvant chemotherapy are useful in predicting the histologic responses of patients with osteosarcoma $(10,18,23)$. Several researchers $(3,8,9)$, however, reported that $\triangle \mathrm{MRV}$ after neoadjuvant chemotherapy was not correlated with histologic response. The lack of correlation between $\triangle \mathrm{MRV}$ and histologic response in the current study may be attributable to the slow regression of the osteoid matrix and cystic degeneration in good responders with a corresponding fallacious increase in tumor volume on MR imaging scans (23). Another possible explanation is the relatively small sample size, which can limit the ability to detect significant differences because of reduced statistical power.

Consistent with our data, previous studies in breast cancer and gastrointestinal stromal tumors have shown a significant negative correlation between $\triangle \mathrm{SUV}$ and $\triangle \mathrm{ADC}(24,25)$. In osteosarcoma with good histologic response after neoadjuvant chemotherapy, at least $90 \%$ of cancer tissue is necrotic and less than $10 \%$ of primary viable cancer cells remain (20). Hence, a decrease in cellularity and a loss of viable cells are postulated to underlie an increase in ADC value and a decrease in SUV, respectively, in good histologic responders (12).

Both PET/CT $(3,9,26)$ and DWI $(2,7,8)$ have been reported to be of value for response assessment in osteosarcoma. Several studies have suggested $-60 \%$ to $-40 \%$ of $\triangle \mathrm{SUV}$ as the cutoff value for good response to neoadjuvant chemotherapy of osteosarcoma $(3,9,26)$. However, increased ${ }^{18}$ F-FDG uptake caused by inflammatory infiltrates or reactive fibrosis within a responding tumor may hamper the monitoring of histologic responses (27). Moreover, the partial-volume effect produced by limited spatial 


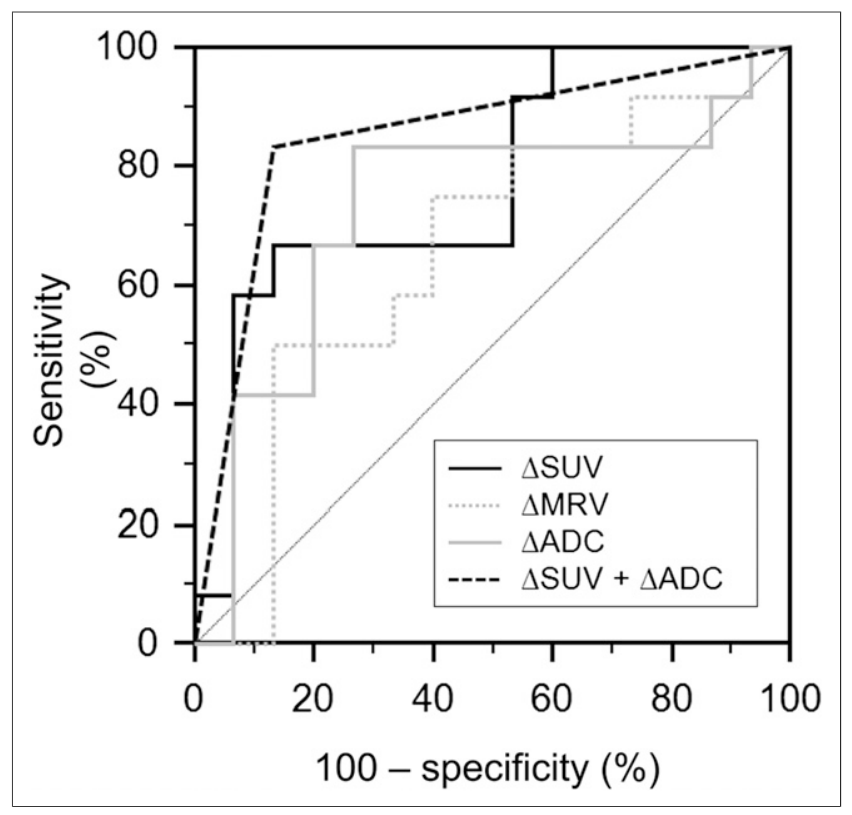

FIGURE 4. ROC curves used to evaluate good response to neoadjuvant chemotherapy with $\triangle S U V, \triangle M R V, \triangle A D C$, and combined use of $\triangle S U V$ and $\triangle A D C$. AUC of $\triangle S U V(0.772)$ was higher than that of $\triangle \mathrm{MRV}(0.656)$ or $\triangle \mathrm{ADC}(0.728)$, but no significant difference was found. AUC increased with combined use of $\triangle S U V$ and $\triangle A D C$ (0.850), but there was no significant difference.

resolution may cause the ${ }^{18} \mathrm{~F}-\mathrm{FDG}$ uptake in small or necrotic lesions to be underestimated (10). Although the optimal cutoff value of $\triangle \mathrm{ADC}$ for good response to neoadjuvant chemotherapy of osteosarcoma was not reported in previous studies, ADC value and its change after chemotherapy have been reported to correlate with histologic response in osteosarcoma $(2,7,8)$. A spin-echo echo-planar imaging sequence was chosen in the current study because it can be performed within a few seconds, reducing the risk of motion artifacts. However, this sequence usually has a relatively low signal-to-noise ratio and is susceptible to artifacts (8). Furthermore, there is no standard method to measure the ADC value (7). The combined use of $\triangle \mathrm{SUV}$ and $\triangle \mathrm{ADC}$ can provide various biologic information (i.e., changes in glucose metabolism and cellularity) and thus may overcome the potential limitations of PET/CT and DWI. The current preliminary results indicate that the integration of PET/CT and DWI may be an effective method to predict patients' histologic responses. On the basis of our results, further studies with large study populations using these 2 imaging modalities may be warranted to evaluate the benefits of their combined use, compared with each modality alone.

In the current study protocol with sequential PET/CT and MR imaging, MR imaging was performed immediately after the completion of PET/CT imaging on the same day. If these examinations were performed hours or days apart, anatomic colocalization or imaging parameters (SUV, ADC, or tumor volume) might have changed because of the regression or progression of osteosarcoma (28). Although the sequential acquisition of PET/CT and MR imaging may give less accurate alignments of both datasets, compared with the simultaneous acquisition, sequential acquisition was sufficient for independently measuring the imaging parameters. A hybrid PET/MR imaging scanner, however, allows the simultaneous acquisition of PET and MR imaging data and, thus, essentially perfect temporal and spatial correlation of acquired datasets from both modalities. With the voxelwise analysis of data from hybrid PET/MR imaging, for example, diagnostic accuracy may be improved (29).

There is no agreed optimal $b$ value for musculoskeletal DWI, and previous studies have used different $b$ values $(0$ and 700 , 0 and $1,000 \mathrm{~s} / \mathrm{mm}^{2}$ ) when the therapeutic responses of patients with osteosarcoma were monitored $(2,3,7,8)$. On the basis of our preliminary experience, the best image quality was obtained with $b$ values of 0 and $800 \mathrm{~s} / \mathrm{mm}^{2}$. Koc et al. (30) reported that the DWIs with $b$ values of 0 and 600, 0 and 800 , and 0 and 1,000 $\mathrm{s} / \mathrm{mm}^{2}$ showed similar abilities in terms of their differentiation between benign and malignant gynecologic lesions. Therefore, we expect that the predictive value of DWI is little affected by the $b$ value used in the present study.

The present preliminary study has several limitations. First, the number of patients was relatively small. Second, an underestimation of SUV because of partial-volume effect in small or necrotic lesions may reduce SUV accuracy (31). Third, there is no standard method for measuring the ADC value of osteosarcoma (7), and we did not compare different methods for measuring ADC. Nonetheless, because tumor sizes were relatively large and we selected an axial slice with the largest tumor diameter on ADC maps, we could readily draw regions of interest and measure the ADC value by consensus of 2 of the authors. Fourth, we did not analyze the cost-effectiveness of the studies. Finally, we did not use other PET or MR imaging parameters, such as total lesion glycolysis $(9,10)$ or other kinetic methods $(3,8)$.

\section{CONCLUSION}

Both PET/CT and DWI are useful for predicting histologic response after neoadjuvant chemotherapy in osteosarcoma. The combined use of PET/CT and DWI may be an effective method to predict histologic response to neoadjuvant chemotherapy.

\section{DISCLOSURE}

The costs of publication of this article were defrayed in part by the payment of page charges. Therefore, and solely to indicate this fact, this article is hereby marked "advertisement" in accordance with 18 USC section 1734. This work was supported by Establishment of Center for PET Application Technology Development, Korea Institute of Radiological and Medical Sciences (KIRAMS), and by grants from the Ministry of Education, Science and Technology (50441-2012). No other potential conflict of interest relevant to this article was reported.

\section{REFERENCES}

1. Bacci G, Longhi A, Fagioli F, Briccoli A, Versari M, Picci P. Adjuvant and neoadjuvant chemotherapy for osteosarcoma of the extremities: 27 year experience at Rizzoli Institute, Italy. Eur J Cancer. 2005;41:2836-2845.

2. Uhl M, Saueressig U, Koehler G, et al. Evaluation of tumour necrosis during chemotherapy with diffusion-weighted MR imaging: preliminary results in osteosarcomas. Pediatr Radiol. 2006;36:1306-1311.

3. Bajpai J, Gamnagatti S, Kumar R, et al. Role of MR imaging in osteosarcoma for evaluation and prediction of chemotherapy response: correlation with histological necrosis. Pediatr Radiol. 2011;41:441-450.

4. Davis AM, Bell RS, Goodwin PJ. Prognostic factors in osteosarcoma: a critical review. J Clin Oncol. 1994;12:423-431.

5. Söderlund V, Larsson SA, Bauer HC, Brosjo O, Larsson O, Jacobsson H. Use of ${ }^{99 m}$ Tc-MIBI scintigraphy in the evaluation of the response of osteosarcoma to chemotherapy. Eur J Nucl Med. 1997;24:511-515. 
6. Wellings RM, Davies AM, Pynsent PB, Carter SR, Grimer RJ. The value of computed tomographic measurements in osteosarcoma as a predictor of response to adjuvant chemotherapy. Clin Radiol. 1994;49:19-23.

7. Oka K, Yakushiji T, Sato H, Hirai T, Yamashita Y, Mizuta H. The value of diffusion-weighted imaging for monitoring the chemotherapeutic response of osteosarcoma: a comparison between average apparent diffusion coefficient andminimum apparent diffusion coefficient. Skeletal Radiol. 2010;39:141-146.

8. Hayashida Y, Yakushiji T, Awai K, et al. Monitoring therapeutic responses of primary bone tumors by diffusion-weighted image: initial results. Eur Radiol. 2006;16:2637-2643.

9. Im HJ, Kim TS, Park SY, et al. Prediction of tumour necrosis fractions using metabolic and volumetric ${ }^{18} \mathrm{~F}$-FDG PET/CT indices, after one course and the completion of neoadjuvant chemotherapy, in children and young adults with osteosarcoma. Eur J Nucl Med Mol Imaging. 2012;39:39-49.

10. Cheon GJ, Kim MS, Lee JA, et al. Prediction model of chemotherapy response in osteosarcoma by ${ }^{18}$ F-FDG PET and MR imaging. J Nucl Med. 2009;50:1435-1440.

11. Schulte M, Brecht-Krauss D, Werner M, et al. Evaluation of neoadjuvant therapy response of osteogenic sarcoma using FDG PET. J Nucl Med. 1999;40:1637-1643.

12. Choi SY, Chang YW, Park HJ, Kim HJ, Hong SS, Seo DY. Correlation of the apparent diffusion coefficiency values on diffusion-weighted imaging with prognostic factors for breast cancer. Br J Radiol. 2012;85:e474-e479.

13. Drzezga A, Souvatzoglou M, Eiber M, et al. First clinical experience with integrated whole-body PET/MR: comparison to PET/CT in patients with oncologic diagnoses. J Nucl Med. 2012;53:845-855.

14. Delso G, Ziegler S. PET/MR imaging system design. Eur J Nucl Med Mol Imaging. 2009;36(suppl 1):S86-S92.

15. Buchbender C, Heusner TA, Lauenstein TC, Bockisch A, Antoch G. Oncologic PET/MR imaging, part 1: tumors of the brain, head and neck, chest, abdomen, and pelvis. J Nucl Med. 2012;53:928-938.

16. Buchbender C, Heusner TA, Lauenstein TC, Bockisch A, Antoch G. Oncologic PET/MR imaging, part 2: bone tumors, soft-tissue tumors, melanoma, and lymphoma. J Nucl Med. 2012;53:1244-1252.

17. Rosen G, Marcove RC, Huvos AG, et al. Primary osteogenic sarcoma: eight-year experience with adjuvant chemotherapy. J Cancer Res Clin Oncol. 1983;106 (suppl):55-67.

18. Kim MS, Lee SY, Cho WH, et al. Tumor necrosis rate adjusted by tumor volume change is a better predictor of survival of localized osteosarcoma patients. Ann Surg Oncol. 2008;15:906-914.
19. Bieling P, Rehan N, Winkler P, et al. Tumor size and prognosis in aggressively treated osteosarcoma. J Clin Oncol. 1996;14:848-858.

20. Coffin CM, Lowichik A, Zhou H. Treatment effects in pediatric soft tissue and bone tumors: practical considerations for the pathologist. Am J Clin Pathol. 2005;123:75-90.

21. Shultz EK. Multivariate receiver-operating characteristic curve analysis: prostate cancer screening as an example. Clin Chem. 1995;41:1248-1255.

22. Hanley JA, McNeil BJ. A method of comparing the areas under receiver operating characteristic curves derived from the same cases. Radiology. 1983;148: $839-843$.

23. van der Woude HJ, Bloem JL, Hogendoorn PC. Preoperative evaluation and monitoring chemotherapy in patients with high-grade osteogenic and Ewing's sarcoma: review of current imaging modalities. Skeletal Radiol. 1998;27: 57-71.

24. Park SH, Moon WK, Cho N, et al. Comparison of diffusion-weighted MR imaging and FDG PET/CT to predict pathological complete response to neoadjuvant chemotherapy in patients with breast cancer. Eur Radiol. 2012;22:1825 .

25. Gong NJ, Wong CS, Chu YC, Gu J. Treatment response monitoring in patients with gastrointestinal stromal tumor using diffusion-weighted imaging: preliminary results in comparison with positron emission tomography/computed tomography. NMR Biomed. 2013;2:185-192.

26. Huang TL, Liu RS, Chen TH, Chen WY, Hsu HC, Hsu YC. Comparison between F-18-FDG positron emission tomography and histology for the assessment of tumor necrosis rates in primary osteosarcoma. J Chin Med Assoc. 2006;69: 372-376.

27. Brenner W, Bohuslavizki KH, Eary JF. PET imaging of osteosarcoma. J Nucl Med. 2003;44:930-942.

28. Werner MK, Schmidt H, Schwenzer NF. MR/PET: a new challenge in hybrid imaging. AJR. 2012;199:272-277.

29. Kinoshita M, Arita H, Goto T, et al. A novel PET Index, ${ }^{18} \mathrm{~F}$-FDG- ${ }^{11} \mathrm{C}$-methionine uptake decoupling score, reflects glioma cell infiltration. J Nucl Med. 2012; 53:1701-1708.

30. Koc Z, Erbay G, Ulusan S, Seydaoglu G, Aka-Bolat F. Optimization of b value in diffusion-weighted MR imaging for characterization of benign and malignant gynecological lesions. J Magn Reson Imaging. 2012;35:650-659.

31. Soret M, Bacharach SL, Buvat I. Partial-volume effect in PET tumor imaging. J Nucl Med. 2007;48:932-945. 